about their practices, is also required. This has been the rationale for reporting hospital infection statistics for many years. Because severity of illness and risk of infection vary greatly among intensive care patients, patient-care practice-performance information may have a greater role on staff behavior than infection rates alone. When medical staff identify the consequences of their performance with patient outcomes, they are more likely to improve. They may also make value judgments on the need for hand decontamination that are not obvious to an observer but are based on the real risk of cross-infection. These factors, guided by case-mix, can confound observational studies, but in general those with more knowledge decontaminate hands more appropriately.

If cross-infections in intensive care units are to be controlled effectively, we must recognize the complex dynamics that change and the conjunction of factors that are commonly responsible for outbreaks. The presence of a multiply resistant bacteria and a low priority for hand disinfection are a recipe for disaster; add a holiday or flu season, resulting in fewer staff, and it is the "too few hands" that ignite the problem.

\section{READING LIST}

Gaynes RP, Solomon S. Improving hospitalacquired infection rates: the CDC experience. J Qual Improv 1996;22:457-467.

Gould D. Nurses' hand decontamination practice: results of a local study. J Hosp Infect 1994;28:15-30.

Peter Heseltine, MD

University of Southern California Los Angeles, California

\title{
Computer-Generated Dot Map Identifies Source of Toxoplasma gondii Outbreak
}

\section{Gina Pugliese, RN, MS Martin S. Favero, $\mathbf{P h D}$}

Computer-generated dot maps were used to examine the spatial distribution of 94 Toxoplasma gondii infections associated with an outbreak in British Columbia, Canada. The incidence among patients served by one water distribution system was found to be 3.52 times that of patients served by other sources. Acute $T$ gondii infection among 3,812 pregnant women was associated with the incriminated distribution system.

Epidemiologists have traditionally used maps to examine the spatial distribution of disease incidence. Because most populations are served by identifiable water systems, waterborne disease outbreaks lend themselves to being plotted on dot maps. The researchers used an automated address-matching and base map system in a geographic information system.

In March 1995, an outbreak of toxoplasmosis was suspected when 15 residents of the district were identified as having acute infection with $T$ gondii. Investigation of these and subsequent cases confirmed an outbreak but identified no common food, beverage, or event source. A handdrawn dot map of the initial 47 cases showed clustering in the Greater Victoria area. The municipal water supply system was considered a possible explanation for this spatial distribution. To examine this hypothesis, computer-based geographic mapping was used to study the distribution of all outbreak-related acute cases and data collected from a population-based serological screening program to detect $T$ gondii infection in women who were or had been pregnant.
The researchers noted that, although there are limitations of geographic mapping analysis of cases, such as inability to adjust for age or other covariates, it has numerous advantages. Advantages include (1) facilitating the verification and correct placement of addresses; (2) reducing the time required to map the location of large data sets; (3) enabling queries and statistical analysis of the data after mapping; (4) allowing several sets of mapped data to be analyzed simultaneously for potential relationships; and (5) generating printouts or overheads for presentations.

FROM: Eng SB, Werker DH, King AS, Marion SA, Bell A, IsaacRenton JL, et al. Computer-generated dot maps as an epidemiologic tool: investigating an outbreak of toxoplasmosis. Emerg Infect Dis 1999;5:815 819. 